Canad. Math. Bull. Vol. 20 (1), 1977

\title{
A KNESER THEOREM FOR HIGHER ORDER ELLIPTIC EQUATIONS
}

\author{
BY \\ W. ALLEGRETTO
}

The problem of establishing oscillation and non-oscillation criteria for elliptic equations has recently been considered by several authors. Extensive bibliographies may be found in the books by C. A. Swanson, [7], and by K. Kreith, [3].

Most of the interest has so far centered on equations of second order with some results also established for fourth order equations. Non-oscillation theorems for higher order equations have recently been established by the author, [1], and by Noussair and Yoshida, [5]. In particular, both in [1] and [5], Kneser-type theorems were established for classes of higher order elliptic equations. However, these results become vacuous in the case where the dimension of the underlying Euclidean space is even and not larger than the order of the operator in question. The establishment of a Kneser-type theorem for such a case is mentioned in [1] as an open question. It is the purpose of this paper to establish a Kneser-type theorem which gives a non-vacuous result for this case. Our theorem is obtained by extending the estimates given in [1].

Let $\Omega$ be an unbounded domain of $m$-dimensional Euclidean space $E^{m}$. Points of $E^{m}$ are denoted by $x=\left(x_{1}, \ldots, x_{m}\right)$ and differentiation with respect to $x_{i}$ by $D_{i}, i=1, \ldots, m$. We consider the differential expression $L$ formally given in the standard multi-index notation by:

$$
L u=(-1)^{n} \sum_{|\alpha|=|\beta|=n} D^{\alpha}\left(a_{\alpha \beta} D^{\beta} u\right)-a_{00} u, \quad a_{\alpha \beta}=a_{\beta \alpha} .
$$

The coefficients of $L$ are defined in $\Omega$, real, and sufficiently regular so that all derivatives involved in $L$ exist and are at least continuous in $\Omega-R$ for some sphere $R$.

As in [1], we term $L$ non-oscillatory iff $L$ has no nodal domains in $\Omega-R$ for some sphere $R$. We assume that there exists an elliptic expression $l_{0}$, given by:

$$
l_{0} u=(-1)^{n} \sum_{|\alpha|=|\beta|=n} D^{\alpha}\left(b_{\alpha \beta} D^{\beta} u\right),
$$

with constant coefficients and ellipticity constant $\mu_{0}$ such that the inequality:

$$
\int_{\Omega} \sum_{|\alpha|=|\beta|=n} a_{\alpha \beta}(x) D^{\alpha} \phi D^{\beta} \phi d x \geq \int_{\Omega} \sum_{|\alpha|=|\beta|=n} b_{\alpha \beta} D^{\alpha} \phi D^{\beta} \phi d x,
$$

Received by the editors June 17, 1976 and, in revised form, Oct. 4, 1976. 
holds for all $\phi \in C_{0}^{\infty}(\Omega \wedge\{|x|>r\})$. It then follows from the results of [1], that for $L$ to be non-oscillatory in $\Omega$ it is sufficient that the expression:

$$
l_{1} u=(-1)^{n} \Delta^{n} u-a_{00} \mu_{0}^{-1} u
$$

be non-oscillatory in $\Omega$. In the sequel, we therefore obtain explicitly our results only for the expression $l_{1}$.

It is clear from the nature of the problem that it is sufficient to consider only subdomains of $\Omega$ which are in the complement of a sufficiently large sphere in $E^{m}$. Consequently, without loss of generality and without further mention we shall always assume that any function $\phi \in C_{0}^{\infty}(\Omega)$ considered in the sequel has support in $\left\{x|| x \mid>^{r}\right\}$ where $r$ is chosen for convenience and sufficiently large.

We begin by stating the following results whose proofs can be found, in particular, in [1] and which will be useful in the sequel.

LemMa 0. (1) Let $t$ be a positive (non-negative, respectively) integer, $m>4 t$, $\left(m>4 t+2\right.$ respectively) then for all $\phi \in C_{0}^{\infty}(\Omega)$ the following inequalities hold:

$$
\begin{gathered}
\int_{E^{m}}\left(\Delta^{t} \phi\right)^{2} \geq 4^{-2 t} \prod_{i=0}^{2 t-1}(m+4 i-4 t)^{2} \int_{E^{m}} \phi^{2}|x|^{-4 t}, \\
\int_{E^{m}} \sum_{i=1}^{m}\left[D_{i}\left(\Delta^{t} \phi\right)\right]^{2} \geq 4^{-(2 t+1)} \prod_{i=0}^{2 t}(m+4 i-4 t-2)^{2} \int_{E^{m}} \phi^{2}|x|^{-4 t-2} ;
\end{gathered}
$$

(2) If $m=2$, then:

$$
\int_{E^{2}} \sum_{i=1}^{2}\left[D_{i} \phi\right]^{2} \geq \frac{1}{4} \int_{E^{2}} \phi^{2}(|x| \ln |x|)^{-2} d x .
$$

Corollary 0. Assume that $q \geq 0, \quad l \geq 0, \quad \beta \leq 0, \quad \beta+l \geq 0$ and let $h \in$ $C_{0}^{\infty}((1, \infty))$. Then the following inequality is valid:

$$
\begin{aligned}
& \int_{1}^{\infty}\left\{r^{3}(\ln r)^{\beta}\left(D^{2} h\right)^{2}+\left(D^{1} h\right)^{2} r(\ln r)^{\beta}\left[q+\frac{l}{\ln r}\right]\right\} d r \geq \int_{1}^{\infty} \frac{h^{2}(\ln r)^{\beta}}{r} \\
& { }^{(1)} \times\left\{\frac{(1-\beta)^{2}(1+q)}{4}(\ln r)^{-2}+\frac{(\beta+l)(2-\beta)^{2}}{4}(\ln r)^{-3}+\frac{(1-\beta)^{2}(3-\beta)^{2}}{16}(\ln r)^{-4}\right\} d r .
\end{aligned}
$$

Proof. The standard substitution $r=e^{t}$ reduces the left hand side of (1) to:

$$
\int_{0}^{\infty} t^{\beta}\left[D^{2} h\right]^{2} d t+\int_{0}^{\infty}\left[D^{1} h\right]^{2}\left\{(1+q) t^{\beta}+(\beta+l) t^{\beta-1}\right\} d t .
$$

Estimating each part of (2) by means of Lemma 2 and 3 of [1] we find that (2) is at least equal to:

$$
\int_{0}^{\infty}\left\{\frac{(1-\beta)^{2}}{4}(1+q) t^{\beta-2}+\frac{(\beta+1)(2-\beta)^{2}}{4} t^{\beta-3}+\frac{(1-\beta)^{2}(3-\beta)^{2}}{16} t^{\beta-4}\right\} h^{2} d t
$$

and the result follows. 
The next Lemma is an extension of some of the results of [1] (where only the case $\beta \equiv 0$ was considered) and [6] (where only the case $\alpha=\beta=0$ was considered). Since the proof parallels that given in [1], [6], and in view of the lengthy calculations involved, the proof is only sketched.

Lemma 1. Let $\alpha, \beta$ be non-positive scalars. Then for all $\phi \in C_{0}^{\infty}(\Omega)$ the following inequality holds:

$$
\int_{E^{m}} r^{\alpha}(\ln r)^{\beta}(\Delta \phi)^{2} d x \geq \int_{E^{m}} \phi^{2} r^{\alpha-4}(\ln r)^{\beta} F(\alpha, \beta, r) d x
$$

where

$$
F(\alpha, \beta, r)=\sum_{i=0}^{4} K_{i}(\ln r)^{-i}
$$

and

$$
\begin{aligned}
K_{0}(\alpha)= & \frac{(4-\alpha-m)^{2}}{16}(m-\alpha)^{2}+\inf _{k \in\{0,1, \ldots\}}[\tau(\alpha, k)], \\
\tau(\alpha, k)= & k(k+m-2)\left\{k^{2}+(m-2) k+\frac{m^{2}-4 m+4 \alpha-\alpha^{2}}{2}\right\} \\
K_{1}(\alpha, \beta)= & -\frac{\beta}{4}\left[(4-\alpha-m)\left(\alpha^{2}+2 m-\alpha m-2 \alpha\right)+4(\alpha-2) k_{1}\left(k_{1}+m-2\right)\right], \\
K_{2}(\alpha, \beta)= & \frac{k_{1}\left(k_{1}+m-2\right)}{2}\left(1-\beta^{2}\right)+\frac{(4-\alpha-m) \beta(\beta-1)(m-\alpha)}{4} \\
& +\frac{(1-\beta)^{2}}{8}\left[8+\alpha^{2}+m^{2}-4 \alpha-4 m\right], \\
K_{3}(\alpha, \beta)= & \frac{\beta}{4}(2-\beta)^{2}(\alpha-2), \\
K_{4}(\alpha, \beta)= & \frac{(1-\beta)^{2}(3-\beta)^{2}}{16},
\end{aligned}
$$

where, for $\beta \neq 0\left(\beta=0\right.$, respectively), $k_{1}$ denotes the largest (smallest, respectively) non-negative integer at which the infimum of $\tau(\alpha, \cdot)$ is achieved.

Proof. Let $\phi \in C_{0}^{\infty}(\Omega)$. Introducing polar coordinates, we obtain:

$$
\int_{E^{m}} r^{\alpha}(\ln r)^{\beta}(\Delta \phi)^{2} d x=\int_{0}^{\infty} \int_{\Phi}(\Delta \phi)^{2} d w r^{\alpha+m-1}(\ln r)^{\beta} d r,
$$

where $\Phi$ denotes the full range of the angular variables and $d w$ denotes the angular component of the volume element. Let $\left\{Y_{i}\right\}_{i=0}^{\infty}$ denote a complete orthonormal system of spherical harmonics and let the order of $Y_{i}$ be $k=k(i)$. 
It follows that:

(3) $\int_{E^{m}} r^{\alpha}(\ln r)^{\beta}(\Delta \phi)^{2} d x=\sum_{0}^{\infty} \int_{0}^{\infty} r^{\alpha+m-1}(\ln r)^{\beta}\left[D^{2} f_{i}+\frac{(m-1)}{r} D^{1} f_{i}\right.$

$$
\left.-\frac{k(k+m-2)}{r^{2}} f_{i}\right]^{2} d r
$$

where $f_{i}=\int_{\Phi} Y_{i} \phi d w$. Motivated by the discussion preceding Theorem 1 of $[6$, p. 93], we introduce the change of variable $f_{i}=r^{\gamma} h_{i}$ with $\gamma=(4-\alpha-m) / 2$, and integrate by parts repeatedly to reduce the right hand side of (3) to:

(4) $\sum_{0}^{\infty} \int_{0}^{\infty}\left\{r^{3}(\ln r)^{\beta}\left(D^{2} h_{i}\right)^{2}+\left(D^{1} h_{i}\right)^{2} r(\ln r)^{\beta}\left[2 \gamma^{2}-2 \gamma+a+\frac{b-2 \beta \gamma}{\ln r}\right]\right.$

$$
\begin{aligned}
& +h_{i}^{2} r^{-1}(\ln r)^{\beta}\left[\left(\gamma^{2}(\gamma-1)^{2}+a \gamma^{2}+c\right)\right. \\
& +\frac{1}{\ln r}\left(-2 \gamma^{2}(\gamma-1) \beta+\gamma^{2} b+d-\gamma a \beta+\gamma(\gamma-1) \beta\right) \\
& \left.\left.+\frac{1}{(\ln r)^{2}}(e-\gamma b \beta+\gamma(\gamma-1)(\beta-1) \beta+\gamma b)\right]\right\} d r
\end{aligned}
$$

where, for convenience we have introduced the following terms:

$$
\begin{aligned}
& a=(m-1)(1-\alpha)+2 k(k+m-2), \\
& b=-\beta(m-1), \\
& c=k^{2}(k+m-2)^{2}+k(k+m-2)(\alpha+m-4)(2-\alpha), \\
& d=k(k+m-2) \beta(6-2 \alpha-m), \\
& e=-k(k+m-2) \beta(\beta-1) .
\end{aligned}
$$

Next, we assume that $r$ is sufficiently large so that the coefficient of $\left(D^{1} h_{i}\right)^{2}$ is non-negative and estimate the terms in (4) which involve derivatives of $h_{i}$ by means of Corollary 0. It follows that (4) is at least equal to:

(5) $\sum_{0}^{\infty} \int_{0}^{\infty} h_{i}^{2} r^{-1}(\ln r)^{\beta}\left\{\left[\gamma^{2}(\gamma-1)^{2}+a \gamma^{2}+c\right]+(\ln r)^{-1}\left[-2 \gamma^{2}(\gamma-1) \beta+\gamma^{2} b+d\right.\right.$

$$
\begin{aligned}
& -\gamma a \beta+\gamma(\gamma-1) \beta]+(\ln r)^{-2}\left[e-\gamma b \beta+4^{-1}(1-\beta)^{2}(1+q)\right. \\
& +\gamma(\gamma-1) \beta(\beta-1)+\gamma b]+(\ln r)^{-3} 4^{-1}(\beta+l)(2-\beta)^{2} \\
& \left.+(\ln r)^{-4} 4^{-2}(1-\beta)^{2}(3-\beta)^{2}\right\} d r
\end{aligned}
$$

where

$$
\begin{aligned}
& l=-\beta(m-1+2 \gamma) \\
& q=2 \gamma^{2}-2 \gamma+a .
\end{aligned}
$$

We observe that $c$ is the only term in (5) which grows as a quartic in $k$, and that 
the term multiplying $h_{i}^{2} r^{-1}(\ln r)^{\beta-1}$ in (5) is a quadratic in $k$ which is monotonically decreasing in $k$ for $k$ non-negative and $\beta \neq 0$. Consequently, if we let $k_{1}$ be the largest non-negative integer at which $\inf _{k \in\{0,1, \ldots\}}\left[\gamma^{2}(\gamma-1)^{2}+a \gamma^{2}+c\right]$ is assumed, it follows that for $r$ sufficiently large, each term of the sum in (5) multiplying $h_{i}^{2}$ is at least as large as the term in the case $k=k_{1}$. Estimating each term of (5) by this lower bound shows that (5) is not less than:

$$
\sum_{0}^{\infty} \int_{0}^{\infty} h_{i}^{2} r^{-1}(\ln r)^{\beta} F(\alpha, \beta, r) d r .
$$

An identical argument shows this result for $\beta=0$.

Finally, we conclude that:

$$
\begin{aligned}
\int_{E^{m}} r^{\alpha}(\ln r)^{\beta}(\Delta \phi)^{2} d x & \geq \sum_{0}^{\infty} \int_{0}^{\infty} f_{i}^{2} r^{-5+\alpha+m}(\ln r)^{\beta} F(\alpha, \beta, r) d r \\
& =\int_{E^{m}} \phi^{2} r^{\alpha-4}(\ln r)^{\beta} F(\alpha, \beta, r) d x
\end{aligned}
$$

and the statement of the Lemma follows.

We observe that unlike previous results, the factor $F(\alpha, \beta, r)$ in (6) is always positive for $r$ large, regardless of the choices of $\alpha, \beta$, and $m$ which will arise. Specifically we find by direct calculation the following results:

COROllary 1. Let $R^{+}$denote the set of non-negative integers. Then:

(i) if $(4-\alpha-m) / 2 \notin R^{+}$and $\beta=0$, then $F(\alpha, 0, r) \geq K_{0}(\alpha)>0$;

(ii) if $(4-\alpha-m) / 2 \in R^{+}$then $k_{1}=(4-\alpha-m) / 2, K_{0}=K_{1}=0$, and

$$
\begin{aligned}
F(\alpha, \beta, r)>\frac{K_{2}(\alpha, \beta)}{(\ln r)^{2}}=\left[(2-\alpha)^{2}(1-\beta)^{2}+(4-\alpha-m) \beta(\beta-1)\right. & \\
& \times(m-\alpha)] \cdot \frac{1}{4(\ln r)^{2}} .
\end{aligned}
$$

We next adopt the convention that a repeated product in which the index runs from a subscript to a smaller superscript has value 1 .

COROllary 2. For all $\phi \in C_{0}^{\infty}(\Omega)$ we have:

(i) if $m=4 i$, then:

$$
\begin{array}{r}
(4 p \geq m) \quad \int_{E^{m}}\left(\Delta^{p} \phi\right)^{2} \geq\left\{\prod_{j=0}^{2 i-3}(2 j+2)^{2}\right\}\left\{\prod_{j=0}^{p-i} K_{2}(4-m-4 j,-2 j)\right\} \\
\cdot \int_{E^{m}} \phi^{2} r^{-4 p}(\ln r)^{2(i-p-1)} ; \\
(4 p+2 \geq m) \quad \int_{E^{m}} \sum_{j=1}^{m}\left\{D_{j}\left[\Delta^{p} \phi\right]\right\}^{2} \geq\left\{\prod_{j=0}^{2 i-2}(2 j+1)^{2}\right\}\left\{\prod_{j=0}^{p^{-i}} K_{2}(2-m-4 j,-2 j)\right\} \\
\cdot \int_{E^{m}} \phi^{2} r^{-4 p-2}(\ln r)^{2(-p+i-1)} ;
\end{array}
$$


(ii) if $m=4 i+2, m \neq 2$, then:

$$
\begin{array}{r}
(4 p \geq m) \quad \int_{E^{m}}\left(\Delta^{p} \phi\right)^{2} \geq\left\{\prod_{j=0}^{2 i-1}(2 j+1)^{2}\right\}\left\{\prod_{j=0}^{p-i-1} K_{2}(-m+2-4 j,-2 j)\right\} \\
\cdot \int_{E^{m}} r^{-4 p}(\ln r)^{2(i-p)} \phi^{2} \\
(4 p+2 \geq m) \quad \int_{E^{m}} \sum_{j=1}^{m}\left\{D_{j}\left[\Delta^{p} \phi\right]\right\}^{2} \geq\left\{\prod_{j=0}^{2 i-2}(2 j+2)^{2}\right\}\left\{\prod_{j=0}^{p-i} K_{2}(4-m-4 j,-2 j)\right\} \\
\cdot \int_{E^{m}} r^{-4 p-2}(\ln r)^{2(i-p-1)} \phi^{2}
\end{array}
$$

(iii) if $m=2$, then:

$$
\int_{E^{2}} \sum_{j=1}^{2}\left\{D_{j}\left[\Delta^{p} \phi\right]\right\}^{2} \geq \frac{1}{4}\left\{\prod_{j=0}^{p-1} K_{2}(-2-4 j,-2-2 j)\right\} \cdot \int_{E^{m}} r^{-2-4 p}(\ln r)^{-2-2 p} \phi^{2} .
$$

Proof. We only give a short proof of the first part of (i), since the other parts of the Corollary are established in a similar fashion from Lemma 0 and 1 . We note that:

$$
\int_{E^{m}}\left(\Delta^{p} \phi\right)^{2}=\int_{E^{m}}\left(\Delta^{i-1}\left[\Delta^{p-i+1} \phi\right]\right)^{2} .
$$

Since $4(i-1)<m$, we employ Lemma 0 to conclude:

$$
\int_{E^{m}}\left(\Delta^{p} \phi\right)^{2} \geq \prod_{j=0}^{2 i-3}(2 j+2)^{2} \int_{E^{m}}\left(\Delta^{p-i+1} \phi\right)^{2} r^{4-m}
$$

Next, by part (ii) of Corollary 1 , it follows that:

$$
\int_{E^{m}}\left(\Delta^{p-i+1} \phi\right)^{2} r^{4-m} \geq K_{2}(4-m, 0) \int_{E^{m}}\left(\Delta^{p-i} \phi\right)^{2} r^{-m}(\ln r)^{-2}
$$

Continuing by iteration, we find

$$
\int_{E^{m}}\left(\Delta^{p-i+1} \phi\right)^{2} r^{4-m} \geq\left\{\prod_{j=0}^{p^{-i}} K_{2}(4-m-4 j,-2 j)\right\} \int_{E^{m}} \phi^{2} r^{-4 p}(\ln r)^{2(i-p-1)}
$$

The proof now follows by combining the above results.

We can now state our Kneser-type theorem:

THeorem 1. Assume $2 n \geq m$ with $m$ even. A sufficient condition for the expression $l_{1}$ to be non-oscillatory is given by:

(i) $(m=4 i, 2 n=4 p)$

$\underset{|x| \rightarrow \infty}{\lim \sup _{0}}\left\{a_{00} \mu_{0}^{-1}|x|^{2 n}(\ln |x|)^{(2 n-m+4) / 2}\right\}<\left\{\prod_{j=0}^{2 i-3}(2 j+2)^{2}\right\} \cdot\left\{\prod_{j=0}^{p-i} K_{2}(4-m-4 j,-2 j)\right\}$; 
(ii) $(m=4 i, 2 n=4 p+2)$

$\limsup _{|x| \rightarrow \infty}\left\{a_{00} \mu_{0}^{-1}|x|^{2 n}(\ln |x|)^{(2 n-m+2) / 2}\right\}<\left\{\prod_{j=0}^{2 i-2}(2 j+1)^{2}\right\} \cdot\left\{\prod_{j=0}^{p-i} K_{2}(2-m-4 j,-2 j)\right\}$

(iii) $(m=4 i+2,2 n=4 p)$

$\limsup _{|x| \rightarrow \infty}\left\{a_{00} \mu_{0}^{-1}|x|^{2 n}(\ln |x|)^{(2 n-m+2) / 2}\right\}<\left\{\prod_{j=0}^{2 i-1}(2 j+1)^{2}\right\}$

$$
\cdot\left\{\prod_{j=0}^{p-i-1} K_{2}(-m+2-4 j,-2 j)\right\}
$$

(iv) $(m=4 i+2,2 n=4 p+2)$

$\underset{|x| \rightarrow \infty}{\limsup }\left\{a_{00} \mu_{0}^{-1}|x|^{2 n}(\ln |x|)^{(2 n-m+4) / 2}\right\}<\left\{\prod_{j=0}^{2 i-2}(2 j+2)^{2}\right\} \cdot\left\{\prod_{j=0}^{p-i} K_{2}(4-m-4 j,-2 j)\right\}$;

(v) $(m=2,2 n=4 p+2)$

$$
\underset{|x| \rightarrow \infty}{\limsup }\left\{a_{00} \mu_{0}^{-1}|x|^{2 n}(\ln |x|)^{n+1}\right\}<\frac{1}{4}\left\{\prod_{j=0}^{p-1} K_{2}(-2-4 j,-2-2 j)\right\} .
$$

Proof. Again we prove only (i), the other parts being similar. If we assume that $l_{1}$ is oscillatory then given any sphere $S$, there exists a bounded domain $F \subset \Omega-S$ and a function $u \in H_{n}^{0}(F)$ such that:

$$
\left(u, l_{1} u\right)=\left(u,(-1)^{n} \Delta^{n} u\right)-\left(a_{00} \mu_{0}^{-1} u, u\right)=0 .
$$

Since by a simple limit argument we conclude that the estimates of Corollary 2 are also valid for $u$, we can use the Corollary to estimate the inner product $\left(u,(-1)^{n} \Delta^{n} u\right)$. This leads to the desired contradiction.

We illustrate the above results by considering the special case of the fourth order expression:

$$
\Delta^{2} u-\frac{a_{00}}{\mu_{0}} a_{00}{ }^{u}=0
$$

in the complement of a sphere in $E^{2}$ and in $E^{4}$. In this case we find that the expression is non-oscillatory if:

$$
\limsup _{|x| \rightarrow \infty}\left[a_{00} \mu_{0}^{-1}|x|^{4}(\ln |x|)^{2}\right]<1
$$

both in $E^{2}$ and $E^{4}$.

\section{REFERENCES}

1. W. Allegretto, Nonoscillation theory of elliptic equations of order 2n, Pacific J. Math., 64 (1976), 1-16.

2. A. Friedman, Partial differential equation, Holt, Rinehart and Winston, Inc., New York 1969. 
3. K. Kreith, Oscillation theory, Lecture Notes in Mathematics, Vol. 324, Springer-Verlag, Berlin 1973.

4. E. S. Noussair, Oscillation theory of elliptic equations of order $2 m$, J. Differential Equations 10 (1971), 100-111. MR43, \#6564.

5. E. Noussair and N. Yoshida, Nonoscillation criteria for elliptic equations of order $2 \mathrm{~m}$, submitted for publication.

6. F. Rellich, Perturbation theory of eigenvalue problems, Gordon and Breach, New York 1969. MR39, \#2014.

7. C. A. Swanson, Comparison and oscillation theory of linear differential equations Academic Press, New York and Londen, 1968.

University of Alberta 